\title{
Studying Milky Way structure using stellar populations
}

\author{
J. T. A. de Jong ${ }^{1}$, D. J. Butler ${ }^{1}$, H-W. Rix ${ }^{1}$ \\ A. E. Dolphin ${ }^{2}$ and D. Martínez-Delgado ${ }^{3}$ \\ ${ }^{1}$ Max-Planck-Institut für Astronomie, Königstuhl 17, 69117 Heidelberg, Germany \\ email: dejong@mpia.de \\ ${ }^{2}$ Steward Observatory, Univ. of Arizona, 933 N. Cherry Ave, Tucson, AZ, 85721, United States \\ ${ }^{3}$ Instituto de Astrofísica de Canarias, C/ Vía Láctea, E38200, La Laguna, Spain
}

\begin{abstract}
With the advent of large wide-field cameras and deep, CCD-based "all-sky" surveys, the study of the structure of the Milky Way (MW) through its stellar populations has become feasible. We are developing tools to apply CMD fitting techniques for this purpose. Here we present two applications. First we constrain the properties of the old and young stellar populations of the Canis Major overdensity. Secondly, we demonstrate how CMD fitting can be used to detect faint overdensities in the halo of the MW and determine their properties, using data from the Sloan Digital Sky Survey (SDSS).
\end{abstract}

Keywords. Galaxy: halo, Galaxy: stellar content, Galaxy: structure, galaxies: dwarf, galaxies: individual (Canis Major), galaxies: stellar content

\section{Introduction}

Stars occupy a very specific location in the luminosity-color plane which is determined by their mass, age and metallicity. This means that the intrinsic properties of a stellar population can be constrained from the location of its stars in a color-magnitude diagram (CMD). The information in a CMD is used optimally when fitting the complete distribution of stars. Such CMD fitting techniques have been succesfully used to determine the properties of nearby globular clusters and dwarf galaxies. Now that large wide-field cameras have become available, as well as large "all sky" surveys, such as SDSS and 2MASS, there is the potential to study the stellar populations of the Milky Way (MW) in the same way. However, since we are located within the MW, its stellar populations are spread out along each line-of-sight, smearing out CMD features to be along the magnitude axis. Even if certain stellar overdensities are constrained to a small distance range, large numbers of fore- and background stars will complicate such a study significantly.

Using an adapted version of the MATCH software (Dolphin 1997; Dolphin 2002) we are developing methods to apply CMD fitting techniques to the study of the MW. The software transforms an observed CMD into a so-called Hess diagram, the 2D density of stars as function of color and magnitude. Theoretical evolutionary tracks (Girardi et al. 2002) and a standard Salpeter initial mass function are convolved with models of the photometric errors and completeness, obtained from artificial star tests, to create model Hess diagrams. Using maximum-likelyhood techniques the best-fitting combination of model populations is determined. The adaptations made to the software include the implementation of distance as one of the parameters that can be solved for and the inclusion of isochrones in SDSS filters (Girardi et al. 2004). Below we describe two applications, namely the study of the Canis Major stellar overdensity and the detection and characterization of substructure in the MW halo using SDSS data. 

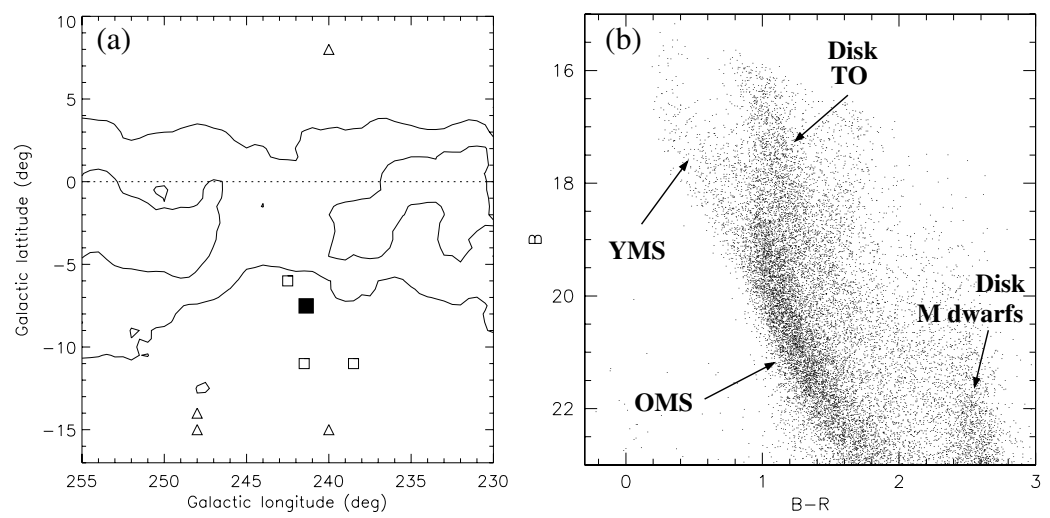

Figure 1. (a) Location of four $1^{\circ} \times 1^{\circ}$ fields used for a CMD fitting analysis of the CMa overdensity (squares). Contours correspond to constant reddenings of $\mathrm{E}(\mathrm{B}-\mathrm{V})=0.4$ and 1.0. (b) $\mathrm{CMD}$ of the field at $(l, b)=(241.5,-7.5)$, indicated with the filled square in (a). The young and old main-sequence features are indicated with YMS and OMS, respectively. Concentrations of thin and thick disk M dwarfs and turn-off (TO) stars are labeled as well.
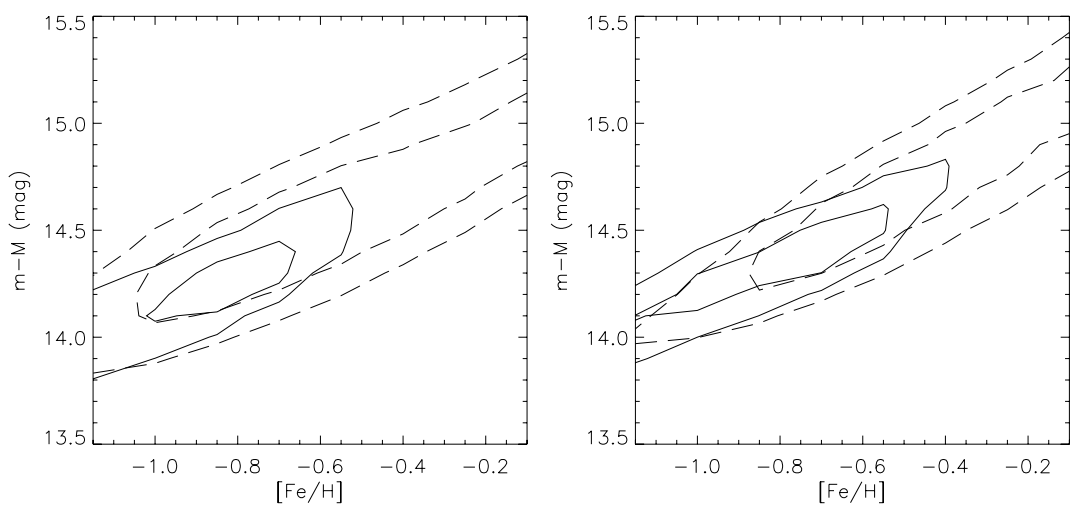

Figure 2. Goodness-of-fit contours $(1$ and $2 \sigma)$ for the fields at $(l, b)=(242.0,-6.0)$ and $(241.5,-7.5)$ as function of metallicity and distance modulus. Solid contours are for the OMS, dashed for the YMS.

\section{The Canis Major overdensity}

In 2004 Martin et al discovered a stellar overdensity in the direction of the constellation Canis Major $(\mathrm{CMa})$, with a suspected center around $(l, b) \simeq(240,-7)$. Both a relatively old $(\sim 6 \mathrm{Gyr})$ and a younger ( $<2$ Gyr) main-sequence feature are seen (OMS and YMS, respectively). Located close to the plane of the disk it is a priori unclear whether or not the overdensity is part of the intrinsic structure of the disk. Different explanations for CMa include the remnant of an accreted dwarf galaxy, an effect of the warp of the outer disk, or out-of-plane spiral arm structure. In the latter case, the YMS stars would be related to the outer Cygnus spiral arm and more distant than the OMS.

Using four fields (Fig. 1a) from a much larger photometric survey of CMa with the Wide Field Imager at the MPG/ESO 2.2m at La Silla (Butler et al. 2007), we have studied the relation between the OMS and YMS. Close the the Galactic plane, the CMDs of these fields contain large numbers of fore- and background stars (Fig. 1b). Both OMS and YMS were fitted separately with model populations with fixed age range, 


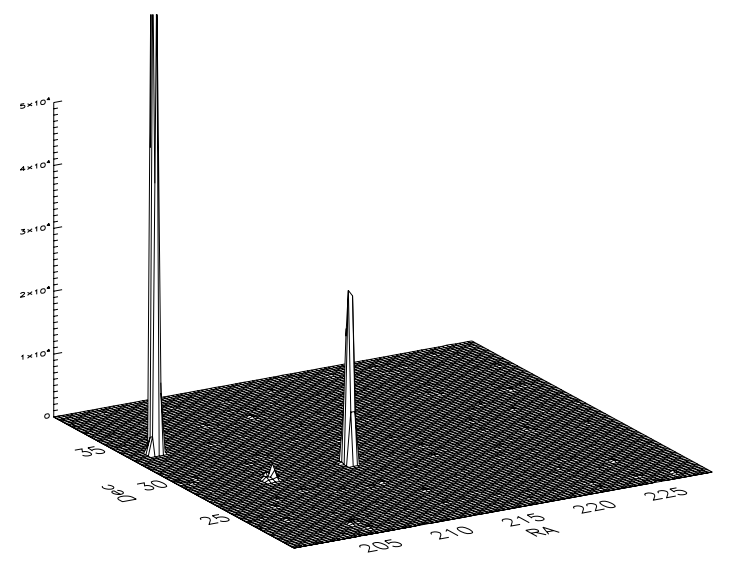

Figure 3. The product of fit quality improvement and number of model stars for $20^{\prime} \times 20^{\prime}$ boxes in a $20^{\circ} \times 20^{\circ}$ field in DR5. The template model used in this case has an age of $10 \mathrm{Gyr}$, $[\mathrm{Fe} / \mathrm{H}]=-2.0$ and distance modulus $\mathrm{m}-\mathrm{M}=21.5$. The peak on the left corresponds to the location of CVnI.
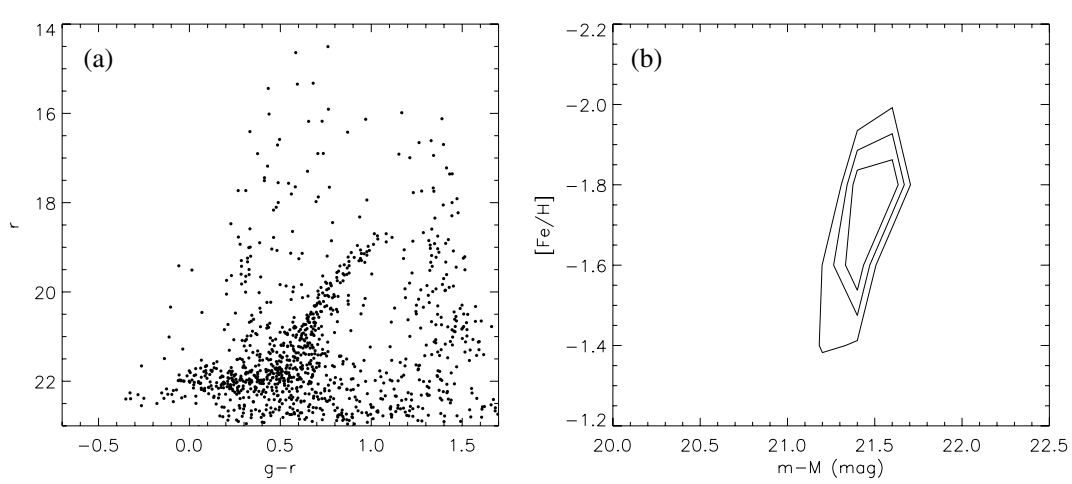

Figure 4. (a) SDSS CMD for the central $0.2^{\circ}$ centered on CVnI. (b) Goodness-of-fit contours $(1,2$, and $3 \sigma)$ as function of distance modulus and metallicity determined for CVnI.

distance, distance spread, and metallicity distribution. Figure 2 shows the results for two fields close to the center of CMa. While the distance and metallicity of the OMS are constrained to be $D \simeq 7.5 \mathrm{kpc}$ and $[\mathrm{Fe} / \mathrm{H}] \simeq-0.8$, the degenaracy between distance and metallicity precludes a robust determination of these parameters for the YMS (de Jong et al., 2007). Two scenarios are consistent with the data: either the YMS has a similar metallicity as the OMS and is equidistant, or the YMS has close to solar metallicity and is more distant $(D \simeq 9 \mathrm{kpc})$.

\section{Halo substructure in SDSS}

The fifth data release (DR5, Adelman-McCarthy et al., 2007) of SDSS covers 8,000 square degrees of sky with photometry down to $r \simeq 23$ and contains approximately 52 million stars, making it the best data set to date for the study of the MW stellar halo. During the past few years several previously unknown stellar overdensities such as tidal streams, dwarf galaxies, and globular clusters have been detected using SDSS data.

CMD fitting techniques are ideal both to detect and to characterize faint stellar overdensities in SDSS data. To detect faint stellar overdensities we create target CMDs for $20^{\prime} \times 20^{\prime}$ boxes and corresponding control field CMDs from $5^{\circ} \times 5^{\circ}$ fields around these 
boxes. Each target CMD is fit by with only the control CMD and with the control CMD plus a simple template stellar population with a fixed age, metallicity and distance. Only if an overdensity with the approximate properties of the template is present in the target box, the improvement in the fit quality and the number of stars contributed to the model population will be significant. Figure 3 shows the product of the number of stars assigned to the model population and the fit improvement for a $\sim 20^{\circ} \times 20^{\circ}$ field. This particular area contains the recently discovered dwarf galaxy Canes Venatici I (CVnI, Zucker et al. 2006), as well as two globular clusters, which all show up as high signal-to-noise (S/N) peaks. The properties of faint overdensities can be determined as well. Figure 4 shows the SDSS CMD (left) of CVnI and goodness-of-fit contours in the distance-metallicity plane (right), determined using similar methods as described in the previous section for CMa. Despite large contamination these properties can be determined very accurately.

\section{Summary}

While CMD fitting techniques to study resolved stellar populations have been used to study isolated objects outside the MW for some time, the application of these techniques to the study of the MW itself is a new field. The reasons for this are the need for deep photometry over large areas of sky, and the fact that stars are spread over a large range of distances. Now that in recent years the former problem has been largely overcome, we show that there is great promise in the study of stellar substructure within the MW using CMD fitting techniques. Application of our software to the deep photometry of the CMa overdensity results in robust estimates of the distance and metallicity of the old stars and shows that the young and old stars might be co-spatial. Using SDSS data for the MW halo shows that these tools can be succesfully used to detect faint overdensities, as well as determine their properties.

\section{Acknowledgements}

JTAdJ and DJB acknowledge support from DFG Priority Program 1177. DMD recognizes the support of the Spanish Ministry of Education and Science (Ramon y Cajal contract and research project AYA 2001-3939-C03-01).

\section{References}

Adelman-McCarthy, et al., 2007, ApJS, subm.

Butler, D. J., Martínez-Delgado, D., Rix, H-W., Peñarrubia, J., \& De Jong, J. T. A., 2007, AJ accepted, astro-ph/0609316

de Jong, J. T. A., Butler, D. J., Rix, H-W., Dolphin, A. E., \& Martínez-Delgado, D., 2007, ApJ, subm., astro-ph/0701140

Dolphin, A. E. 1997, New Astron., 2, 397

Dolphin, A. E. 2002, MNRAS, 332, 91

Girardi, L., Bertelli, G., Bressan, A., Chiosi, C., Groenewegen, M. A. T., Marigo, P., Salasnich, B. \& Weiss, A., 2002, A\& A, 391, 195

Girardi, L., Grebel, E. K., Odenkirchen, M., \& Chiosi, C., 2004, A\&AA, 422, 205

Martin, N. F., Ibata, R. A., Bellazzini, M., Irwin, M. J., Lewis, G. F., \& Dehnen, W., 2004a, MNRAS, 348, 12

Zucker, D. B., Belokurov, V., Evans, N. W., Wilkinson, M. I., Irwin, M. J., Sivarani, T., Hodgkin, S., et al., 2006, ApJ, 643, L103

\section{Discussion}

Ferguson: From your CMD-fitting of the Canis Major overdensity, do you think you are any closer to understanding the origin of the structure?. 
DE JONG: The fact that we are not really sure what the metallicity and distance is of the younger population complicates the interpretation. The metallicity of the old MS stars maybe a bit lower than you would expect for disk but only marginally. We really need spectroscopy; CMD-fitting alone is not decisive.

DUFFAU: Have you checked other overdensities using your technique like overdensities in the field of streams or near Sagittarius like the Virgo stellar stream or the Virgo overdensity?.

DE JONG: No, but we are planning to do so.

Kroupa: Unresolved multiple stellar populations (e.g., binaries) are brighter by up to a factor of 2 than a single star. Have you taken this into account when determining distances using photometric parallax?.

DE JONG: We do account for binaries in our model populations, but have not looked in detail at the effect of using different binary fractions. This would, however, be a good thing to do.

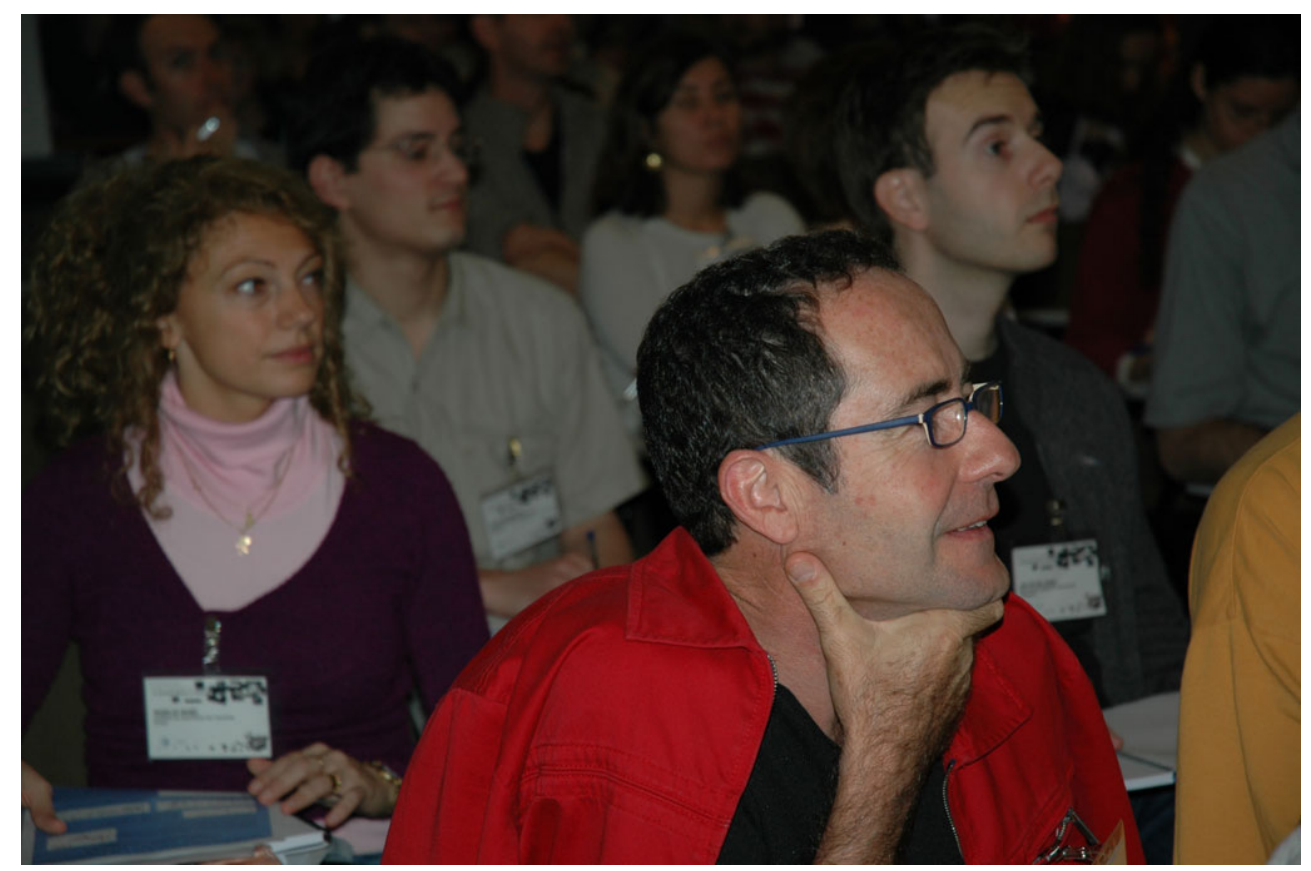

Second row: Noelia Noël and the speaker. First row: Ignacio Garcia de la Rosa. 